\title{
Neurodegenerative Patterns of Cognitive Clusters of Early-Onset Alzheimer's Disease Subjects: Evidence for Disease Heterogeneity
}

\author{
Meredith L. Phillips ${ }^{a}$ Eddie C. Stage, Jr. ${ }^{b, c}$ Kathleen A. Lane ${ }^{c, d}$ \\ Sujuan Gaoc, d Shannon L. Risacher ${ }^{c, e}$ Naira Goukasian ${ }^{f}$ \\ Andrew J. Saykin c, e, g, h Maria C. Carrillo ${ }^{i}$ Bradford C. Dickerson ${ }^{j}$ \\ Gil D. Rabinovicik Liana G. Apostolovab, c, e, g, h \\ Alzheimer's Disease Neuroimaging Initiative
}

\begin{abstract}
a Department of Epidemiology and Biostatistics, Indiana University School of Public Health, Bloomington, IN, USA; ${ }^{b}$ Department of Neurology, Indiana University School of Medicine, Indianapolis, IN, USA; ' Indiana Alzheimer Disease Center, Indiana University School of Medicine, Indianapolis, IN, USA; ${ }^{d}$ Department of Biostatistics, Indiana University School of

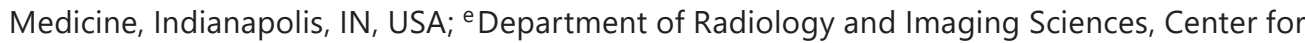
Neuroimaging, Indiana University School of Medicine, Indianapolis, IN, USA; ${ }^{\dagger}$ Larner College of Medicine, Burlington, VT, USA; 9 Department of Medical and Molecular Genetics, Indiana University School of Medicine, Indianapolis, IN, USA; ${ }^{h}$ Indiana University Network Science Institute, Indianapolis, IN, USA; 'Alzheimer's Association, Chicago, IL, USA; ${ }^{j}$ Harvard Medical School, Boston, MA, USA; ${ }^{2}$ University of California San Francisco, San Francisco, CA, USA
\end{abstract}

\section{Keywords}

Early-onset Alzheimer's disease · Cognition · Heterogeneity · Magnetic resonance imaging · Positron emission tomography $\cdot$ Cognitive patterns

\begin{abstract}
Background/Aims: Alzheimer's disease (AD) with onset before 65 (early-onset AD [EOAD]) occurs in approximately $6 \%$ of cases and can affect nonmemory domains. Here, we analyze patterns of impairment in amnestic EOAD individuals using data-driven statistical analyses. Methods: Cognitive data of 146 EOAD subjects were Z-normalized to 395 cognitively normal (CN) individuals. Domain-averaged Z-scores were adjusted for age, sex, and education followed by Wald cluster analysis of residuals. Magnetic resonance imaging and positron emission tomography comparisons of EOAD clusters to age-matched $C N$ were done using Statistic Parametric Mapping 8. Cluster-level-family-wise error $(p<0.05)$ correction was applied.
\end{abstract}

Data used in preparation of this article were obtained from the Alzheimer's Disease Neuroimaging Initiative (ADNI) database (adni.loni.usc.edu). As such, the investigators within the ADNI contributed to the design and implementation of ADNI and/or provided data but did not participate in analysis or writing of this report. 
Mixed-effect models were used to compute longitudinal change across clusters. Results: Scree plot using the pseudo-T-squared suggested a 4-cluster solution. Cluster 1 (memorypredominant impairment) showed atrophy/hypometabolism in medial/lateral temporal, lateral parietal, and posterior cingulate regions. Cluster 2 (memory/visuospatial-predominant) showed atrophy/hypometabolism of medial temporal, temporoparietal, and frontal cortices. Cluster 3 (memory, language, and executive function) and Cluster 4 (globally impaired) manifested atrophy and hypometabolism throughout the brain. Longitudinally between-cluster differences in the visuospatial and language/executive domains were significant, suggesting phenotypic variation. Conclusion: We observed significant heterogeneity in cognitive presentation among amnestic EOAD subjects and patterns of atrophy/hypometabolism in each cluster in agreement with the observed cognitive phenotype.

(c) 2020 S. Karger AG, Basel

\section{Introduction}

In the United States, an estimated 5.5 million people are diagnosed with Alzheimer's disease (AD) - the leading cause of dementia worldwide [1]. Approximately $94 \%$ of patients with $\mathrm{AD}$ become symptomatic after age 65 (late-onset $\mathrm{AD}$ [LOAD]) and 6\% before age 65 (early onset $\mathrm{AD}[\mathrm{EOAD}]$ ] [2]. It has been shown that EOAD patients have more diverse disease presentations and more aggressive disease courses [3-24]. While many LOAD cases have stereotypic memory-predominant deficits, EOAD patients often have atypical presentations with nonmemory impairments early in the disease course [3, 5-9, 11-17, 24]. As many as $64 \%$ of EOAD versus only $12.5 \%$ of LOAD patients present with focal nonamnestic symptoms [6].

Several studies have investigated the heterogeneity of EOAD compared to LOAD. Some studies $[7,9,16]$ reported that, after memory, the language domain is most severely affected in EOAD, while visuospatial function is preserved; others indicate that praxis and visuospatial function are most affected [15, 25]. This inconsistency between EOAD presentations seems to suggest high disease heterogeneity.

We aimed to use a data-driven approach to improve our understanding of the phenotypic heterogeneity among EOAD patients in the AD Neuroimaging Initiative (ADNI) cohort. As ADNI requires all cognitively impaired individuals to manifest significant memory impairment at baseline, our analyses were limited to the amnestic EOAD subtype. We hypothesized that we would find several cognitive subtypes within the amnestic EO group and that the subsequent patterns of neurodegeneration should correspond closely to their respective cognitive phenotype. Furthermore, we hypothesized that amyloid deposition would be similarly diffuse regardless of cognitive subtype. Longitudinal cognitive differences between and within clusters were also analyzed.

\section{Materials and Methods}

Subjects

Data used for this analysis were obtained from the ADNI database (adni.loni.usc.edu). ADNI was launched in 2003 as a public-private partnership, led by Principal Investigator Michael W. Weiner, MD. The primary goal of ADNI has been to test whether serial magnetic resonance imaging (MRI), positron emission tomography (PET), other biological markers, and clinical and neuropsychological assessments can be combined to measure the progression of mild cognitive impairment (MCI) and early AD.

The clinical description of the ADNI cohort has been described previously [26-28]. Diagnosis of AD was based on the National Institute of Neurological and Communicative Disorders and Stroke and the AD and Related Disorders Association criteria [29-31]. The full list of inclusion/exclusion criteria may be accessed 
on pages 23-29 of the online ADNI protocol (see http://www.adni-info.org/Scientists/ADNIScientistsHome. aspx). Written informed consent was obtained from all participants.

The ADNI cohort includes 146 subjects diagnosed with MCI or probable AD with an age of symptom onset $<65$. Of these, $143 \mathrm{had}$ at least one imaging modality available. Our comparison group consisted of 395 cognitively normal (CN) subjects who had GWAS data available and were between the ages of 55 and 80 .

\section{MRI and PET Acquisition and Analyses}

The protocols for MRI image acquisition and preprocessing can be found on www.adni-info.org. ADNI MRI data acquisition and preprocessing have been described elsewhere [32-34]. We downloaded the preprocessed MRI data from LONI IDA (https://ida.loni.usc.edu). All scans were analyzed using voxel-based morphometry using Statistic Parametric Mapping 8, as previously described [35, 36]. Scans were downloaded in NifTI format, coregistered to Montreal Neurological Institute (MNI) space and segmented into gray matter (GM), white matter, and cerebrospinal fluid. The GM maps were normalized to MNI space at $1 \times 1 \times 1 \mathrm{~mm}$ voxel resolution and smoothed using a $10 \mathrm{~mm}$ full-width half maximum Gaussian kernel resulting in GM density (GMD) maps. The intracranial volume was extracted for each subject using FreeSurfer version $5.1[37,38]$.

The F18-fluorodeoxyglucose PET (FDG PET) and 18F-florbetapir PET scan acquisition and preprocessing protocols are available at www.adni-info.org. Scans were obtained using scanners and related equipment that were calibrated and standardized, as described previously [39]. Preprocessed scans were downloaded from LONI IDA (https://ida.loni.usc.edu). The downloaded scans were already averaged, aligned to standard space, resampled to a standard image and voxel size, and smoothed to a uniform resolution [39]. Each image was aligned to the corresponding MRI scan and normalized to MNI space using parameters from the MRI segmentation with $2 \times 2 \times 2 \mathrm{~mm}$ resolution [39]. FDG PET scans were intensity normalized to the pons and the Florbetapir PET scans were intensity normalized to the whole cerebellum to create standard uptake volume ratios (SUVR) images as previously described [39]. These images were then used for voxelwise analysis as described below.

We used the mean amyloid whole-brain SUVR from the University of California Berkeley downloaded from ADNI's database (http://adni.loni.usc.edu). The University of California Berkeley protocols for 18F-florbetapir preprocessing, coregistration, and normalization have been previously described [40].

\section{Statistical Analyses}

Cluster Analysis

Baseline cognitive data from the 146 EOAD subjects meeting criteria for $\mathrm{MCI}$ or dementia were Z-normalized using the mean and SD of the cognitive performance of the $395 \mathrm{CN}$ participants. Neuropsychological tests from the ADNI battery were grouped in 4 domains - memory, language, visuospatial, and executive. The memory domain included the Wechsler Logical Memory Story Recall [41] and the Rey Auditory Verbal Learning Tests (RAVLT) [42]. For the RAVLT, we computed percent learned, which was derived by dividing the total score achieved at trial 5 by the total possible score (15) and multiplying by 100, and RAVLT percent retained, calculated by dividing the score after a 30 -min delay by the score achieved after trial 5 , multiplied by 100. The language domain included the Boston Naming test [43] and animal fluency [44]. The visuospatial domain included clock drawing and clock copy [45]. The executive domain included Trailmaking A and B [46]. For the EOAD participants, all included individuals had complete test data except for a single subject, who was missing a single clock copy score. Domain averaged Z-scores were produced and adjusted for age, sex, and education. Cluster analysis using the Ward method on the residuals for the 4 cognitive domains was performed [47]. Scree plot using the pseudo T-squared was used to determine the optimal number of clusters.

Baseline Demographic and Cognitive Comparisons

Baseline clinical and demographic between-cluster comparisons were conducted using analysis of variance for continuous outcomes and chi-square tests for categorical outcomes.

Imaging Analyses

Using Statistic Parametric Mapping 8, we ran voxel-wise regression analyses to visualize the pattern of GMD, FDG PET, and amyloid PET differences between each EOAD cluster and CN. EOAD cluster was used as the predictor variable with age, sex, and education as covariates. Intracranial volume and MRI field strength were also used as covariates for the MRI analyses. Cluster-level family-wise error (FWE) correction for multiple comparisons was applied. Only clusters surviving FWE correction at the threshold of $p<0.05$ with a minimum cluster size $(\mathrm{k})$ equal to the smallest significant cluster size were displayed. 
Table 1. Demographic and Z-normalized cognitive data (top) and longitudinal data and estimated change and $95 \%$ CIs in cognitive scores adjusting for age, sex, and education over 60 months of follow-up (bottom)

\begin{tabular}{|c|c|c|c|c|c|}
\hline Variable & $\begin{array}{l}\text { Cluster } 1 \\
(n=64)\end{array}$ & $\begin{array}{l}\text { Cluster } 2 \\
(n=31)\end{array}$ & $\begin{array}{l}\text { Cluster } 3 \\
(n=37)\end{array}$ & $\begin{array}{l}\text { Cluster } 4 \\
(n=14)\end{array}$ & $p$ value \\
\hline Number MCI/dementia & $51 / 13$ & $18 / 13$ & $9 / 28$ & $0 / 14$ & $<0.001$ \\
\hline Age, years, mean (SD) & $63.7(4.4)$ & $65.1(6.1)$ & $64.8(6.2)$ & $63.1(5.6)$ & 0.49 \\
\hline Age of symptom onset, years, mean (SD) & $59.2(4.5)$ & $59.3(5.1)$ & $59.5(4.1)$ & $58.3(3.6)$ & 0.87 \\
\hline Gender, male, $\%$ & 51.6 & 45.2 & 45.9 & 57.1 & 0.84 \\
\hline Education, years, mean (SD) & $16.4(2.8)$ & $15.8(2.4)$ & $15.9(3.1)$ & $16.0(2.9)$ & 0.76 \\
\hline Disease duration, years, mean (SD) & $4.5(3.5)$ & $5.8(4.9)$ & $5.3(4.4)$ & $4.8(3.0)$ & 0.51 \\
\hline Amyloid PET SUVR, mean (SD) & $1.26(0.23)$ & $1.33(0.26)$ & $1.34(0.23)$ & $1.48(0.09)$ & 0.091 \\
\hline$\%$ APOE $\varepsilon 4,0 / 1 / 2$ allele(s) & $34 / 39 / 27$ & $23 / 45 / 32$ & $38 / 32 / 30$ & $36 / 43 / 21$ & 0.86 \\
\hline Baseline MMSE, mean (SD) & $27.0(2.1)$ & $25.8(3.1)$ & $24.2(2.5)$ & $21.9(1.7)$ & $<0.001$ \\
\hline Baseline CDRSum of boxes, mean (SD) & $2.0(1.2)$ & $2.9(1.9)$ & $3.9(1.9)$ & $5.5(1.6)$ & $<0.001$ \\
\hline$\%$ of $\mathrm{N}$ with MRI/FDG/amyloid scans & $95.3 / 79.7 / 78.1$ & 87.1/90.3/83.9 & $97.3 / 62.2 / 59.5$ & $92.9 / 78.6 / 50.0$ & - \\
\hline Memory domain, Z-scores, mean (SD) & $-1.77(0.95)$ & $-2.07(1.02)$ & $-2.32(0.75)$ & $-2.89(0.57)$ & $<0.001$ \\
\hline Language domain, Z-scores, mean (SD) & $-0.35(0.87)$ & $-0.99(0.74)$ & $-1.82(1.51)$ & $-2.76(1.84)$ & $<0.001$ \\
\hline Visuospatial domain, Z-scores, mean (SD) & $0.22(0.43)$ & $-2.91(1.88)$ & $-0.96(0.97)$ & $-6.86(2.08)$ & $<0.001$ \\
\hline \multirow[t]{2}{*}{ Executive domain, Z-scores, mean (SD) } & $0.01(0.70)$ & $0.71(1.37)$ & $3.56(1.80)$ & $7.45(2.30)$ & $<0.001$ \\
\hline & $\begin{array}{l}\text { Cluster } 1 \\
(n=64)\end{array}$ & $\begin{array}{l}\text { Cluster } 2 \\
(n=31)\end{array}$ & $\begin{array}{l}\text { Cluster } 3 \\
(n=37)\end{array}$ & $\begin{array}{l}\text { Cluster } 4 \\
(n=14)\end{array}$ & $\begin{array}{l}\text { Time }{ }^{*} \text { cluster } \\
\text { interaction, } \\
p \text { value }\end{array}$ \\
\hline Memory & $\begin{array}{l}-0.077 \\
(-0.47 \text { to } 0.31)\end{array}$ & $\begin{array}{l}0.58 \\
(-0.09 \text { to } 1.24)\end{array}$ & $\begin{array}{l}0.37 \\
(-0.51 \text { to } 1.24)\end{array}$ & $\begin{array}{l}-1.91 \\
(-4.59 \text { to } 0.76)\end{array}$ & 0.15 \\
\hline Language & $\begin{array}{l}-0.84 \\
(-1.29 \text { to }-0.39)\end{array}$ & $\begin{array}{l}-0.10 \\
(-0.86 \text { to } 0.65)\end{array}$ & $\begin{array}{l}-0.40 \\
(-1.45 \text { to } 0.64)\end{array}$ & $\begin{array}{l}-4.87 \\
(-7.81 \text { to }-1.94)\end{array}$ & 0.013 \\
\hline Visuospatial & $\begin{array}{l}-1.60 \\
(-2.26 \text { to }-0.93)\end{array}$ & $\begin{array}{l}1.53 \\
(0.38 \text { to } 2.68)\end{array}$ & $\begin{array}{l}-3.11 \\
(-4.66 \text { to }-1.56)\end{array}$ & $\begin{array}{l}-3.88 \\
(-8.46 \text { to } 0.71)\end{array}$ & $<0.001$ \\
\hline Executive & $\begin{array}{l}1.68 \\
(1.00 \text { to } 2.37)\end{array}$ & $\begin{array}{l}0.25 \\
(-0.92 \text { to } 1.41)\end{array}$ & $\begin{array}{l}1.21 \\
(-0.39 \text { to } 2.80)\end{array}$ & $\begin{array}{l}3.61 \\
(-1.45 \text { to } 8.67)\end{array}$ & 0.16 \\
\hline Number of longitudinal visits, $\%$ & 299 & 114 & 99 & 31 & - \\
\hline 6-month visit & 98.4 & 96.8 & 94.6 & 85.7 & - \\
\hline 12-month visit & 93.8 & 90.3 & 78.4 & 85.7 & - \\
\hline 18-month visit & 31.3 & 12.9 & 5.4 & 0.0 & - \\
\hline 24-month visit & 82.8 & 67.7 & 54.1 & 50.0 & - \\
\hline 36-month visit & 70.3 & 45.2 & 16.2 & 0.0 & - \\
\hline 48-month visit & 59.4 & 38.7 & 10.8 & 0.0 & - \\
\hline 60-month visit & 31.3 & 16.1 & 8.1 & 0.0 & - \\
\hline Months of follow-up, mean (SD) & $41.44(18.08)$ & $32.90(18.72)$ & $21.16(15.60)$ & $17.14(7.12)$ & - \\
\hline
\end{tabular}

Bold values indicate cognitively significant decline and significant $p$ values.

MCI, mild cognitive impairment; MMSE, mini-mental state exam; CDR, clinical dementia rating; PET, positron emission tomography; SUVR, Standard Uptake Volume Ratios; FDG, F18-fluorodeoxyglucose; MRI, magnetic resonance imaging.

Longitudinal Analyses of Cognitive Decline

Longitudinal cognitive data were available on 140 of the 146 subjects. Follow-up varied between 6 months and 10 years. Due to reduction in sample sizes after 60 months, we limited our analyses at 5 years (Table 1). Longitudinal rates of decline in each cognitive domain were examined and compared between the clusters using mixed effects models with repeated measures on all cognitive outcomes after adjusting for education, age, and sex using SAS 9.4. The models included an interaction between time of evaluation from baseline treated as a continuous variable and cluster. The results were visualized by deriving predicted means and 95\% CIs and plotting these for each cluster over time. 
Dementia

and Geriatric

Cognitive Disorders
Dement Geriatr Cogn Disord 2019;48:131-142 DOI: $10.1159 / 000504341$

(c) 2020 S. Karger AG, Basel
www.karger.com/dem

Phillips et al.: Neurodegenerative Patterns of Cognitive Clusters of EOAD Subjects: Evidence for Disease Heterogeneity

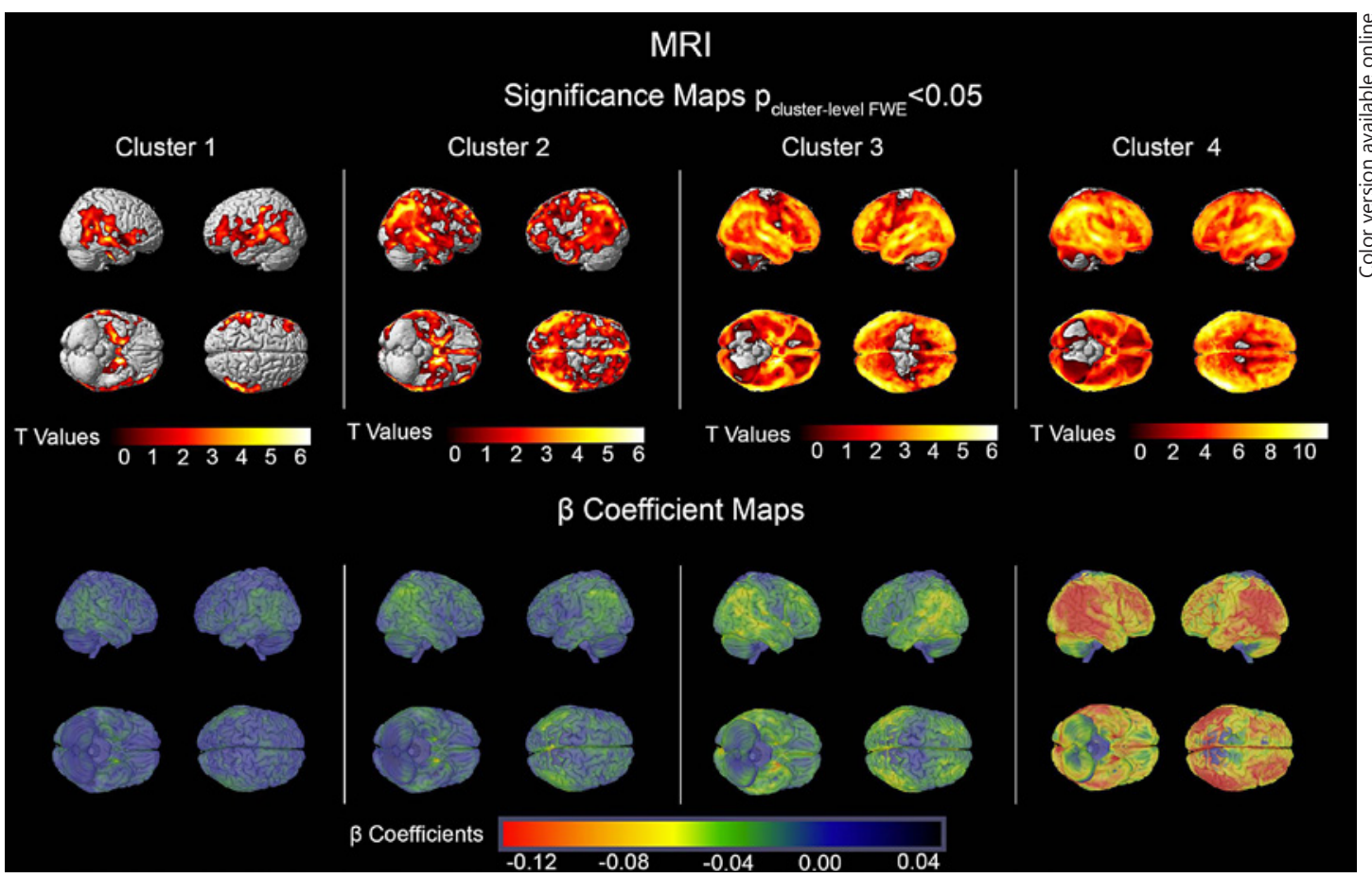

Fig. 1. MRI significance and $\beta$ coefficient maps showing the comparison of each cluster to CN participants. The significance maps show $p<0.05$ thresholded FWE cluster-level corrected results. MRI, magnetic resonance imaging; FWE, family-wise error.

\section{Results}

\section{Baseline Cognitive and Imaging Analyses}

The scree plot suggested a 4-cluster solution. As seen in Table 1, Cluster 1 was comprised of 64 subjects ( $51 \mathrm{MCI}$ and 13 dementia) with isolated memory deficits $(Z=-1.77 \pm 0.95)$. Cluster 2 contained 31 subjects (18 MCI and 13 dementia) with memory and visuospatial domain deficits $\left(\mathrm{Z}_{\text {memory }}=-2.07 \pm 1.02\right.$ and $\left.\mathrm{Z}_{\text {visuospatial }}=-2.91 \pm 1.88\right)$. Cluster 3 had $37 \mathrm{in}$ dividuals (9 MCI and 28 dementia) with impairment in memory, language, and executive function $\left(\mathrm{Z}_{\text {memory }}=-2.32 \pm 0.75, \mathrm{Z}_{\text {language }}=-1.82 \pm 1.51\right.$, and $\left.\mathrm{Z}_{\text {executive }}=3.56 \pm 1.80\right)$. For cluster 4 , there were 14 subjects ( 14 dementia) with deficits in all 4 domains $\left(Z_{\text {memory }}=-2.89 \pm 0.57\right.$, $\mathrm{Z}_{\text {language }}=-2.76 \pm 1.84, \mathrm{Z}_{\text {visuospatial }}=-6.86 \pm 2.08$, and $\mathrm{Z}_{\text {executive }}=7.45 \pm 2.30$ ).

There were no significant between-cluster differences in age, gender, education, APOE4 genotype distribution, age at symptom onset, disease duration, or mean amyloid SUVR. There was a significant between-cluster difference in baseline clinical dementia rating (CDR) and mini-mental state exam, with cluster 1 performing the best and cluster 4 the worst $(p<0.001$ for both). The distribution of diagnoses between the 4 clusters was also significant $(p<0.001)$. The number of subjects used in each imaging analysis varies slightly due to image availability (Table 1).

The MRI and FDG PET imaging comparisons of each cluster relative to the CN sample can be seen in Figures 1, 2, respectively. Cluster 1 showed isolated memory impairment and reduced GMD across the temporal, parietal, and frontal lobes with cluster peaks in the left medial temporal lobe (MTL; $\mathrm{p}_{\mathrm{FWE}}<0.001, \mathrm{k}=86,842$ voxels), the right temporal cortex $\left(\mathrm{p}_{\mathrm{FWE}}<\right.$ $0.001, \mathrm{k}=87,521$ ), and the left and right posterior cingulate cortices (single cluster $\mathrm{p}_{\mathrm{FWE}}=$ $0.002, \mathrm{k}=5,882$ ). They also showed reduced brain metabolism in the MTL (left: $\mathrm{p}_{\mathrm{FWE}}=0.002$, 
Dementia

and Geriatric

Cognitive Disorders
Dement Geriatr Cogn Disord 2019;48:131-142 DOI: $10.1159 / 000504341$

(c) 2020 S. Karger AG, Basel
www.karger.com/dem

Phillips et al.: Neurodegenerative Patterns of Cognitive Clusters of EOAD Subjects: Evidence for Disease Heterogeneity

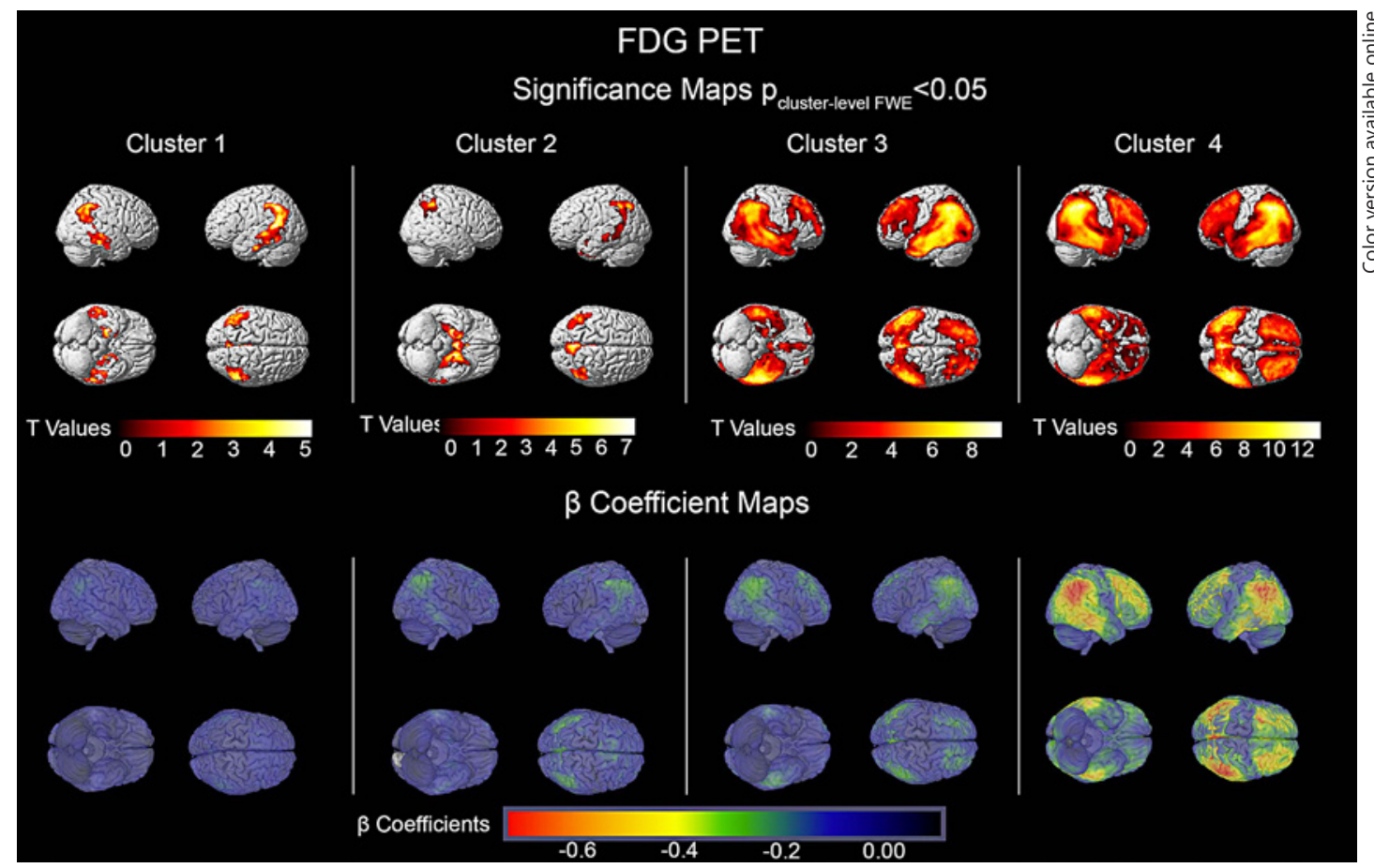

Fig. 2. $\left[{ }^{18} \mathrm{~F}\right]$ FDG PET significance and $\beta$ coefficient maps showing the comparison of each cluster to $\mathrm{CN}$ participants. The significance maps show $p<0.05$ thresholded FWE cluster-level corrected results. FDG PET, F18-fluorodeoxyglucose positron emission tomography; FWE, family-wise error.

$\mathrm{k}=722$; right: $\mathrm{p}_{\mathrm{FWE}}=0.013, \mathrm{k}=494$ ), the bilateral parietal (both $\mathrm{p}_{\mathrm{FWE}}<0.001, \mathrm{k}_{\mathrm{left}}=2,865$, $\left.\mathrm{k}_{\text {right }}=1,133\right)$, and the right temporal cortex $\left(\mathrm{p}_{\mathrm{FWE}}=0.003, \mathrm{k}=688\right)$.

Cluster 2 subjects demonstrated impaired memory and visuospatial function (Table 1). Compared to the $\mathrm{CN}$ group they showed significant reduction in GMD in one large cluster spanning the bilateral MTLs, parietal, and frontal lobes with peak in the left MTL $\left(\mathrm{p}_{\mathrm{FWE}}<0.001, \mathrm{k}=573,388\right)$. Cluster 2 showed hypometabolism in the left MTL $\left(\mathrm{p}_{\mathrm{FWE}}<\right.$ $0.001, \mathrm{k}=14,309$ ) as well as the left and right parietal cortices (left: $\mathrm{p}_{\mathrm{FWE}}<0.001, \mathrm{k}=1,457$; right: $\mathrm{p}_{\mathrm{FWE}}=0.002, \mathrm{k}=736$ ).

Cluster 3 subjects had impaired memory, language, and executive function (Table 1). They showed reduced GMD throughout the brain sparing only portions of the sensorimotor cortex. The GMD cluster peak localized to the left temporal lobe ( $\mathrm{p}_{\mathrm{FWE}}<0.001, \mathrm{k}=1,299,556$ ). Impaired glucose metabolism was seen in the temporal, parietal, and frontal lobes with peak in the fusiform gyrus ( $\mathrm{p}_{\mathrm{FWE}}<0.001, \mathrm{k}=63,532$ ).

Cluster 4 individuals had impairment across all cognitive domains (Table 1). The GMD neurodegeneration spanned the entire brain including the sensorimotor cortices (peak in the right temporal cortex, $\mathrm{p}_{\mathrm{FWE}}<0.001, \mathrm{k}=1,471,637$ ). There was also a global reduction in glucose metabolism sparing the sensorimotor cortices (peak in the left posterior cingulate cortices $\left.\mathrm{p}_{\mathrm{FWE}}<0.001, \mathrm{k}=97,037\right)$. Overall, the largest effect sizes were seen in cluster 4 for both GMD and brain metabolism (Fig. 1, 2, bottom rows). There were no regional differences in amyloid distribution between the clusters (Fig. 3). While cluster 4 showed the highest and cluster 1 the lowest mean cortical amyloid SUVR, the difference only reached a trend level in the analysis of variance analysis ( $p=0.091$, Table 1$)$. 
Dementia

and Geriatric

Cognitive Disorders
Dement Geriatr Cogn Disord 2019;48:131-142 DOI: 10.1159/000504341

(c) 2020 S. Karger AG, Basel
www.karger.com/dem

Phillips et al.: Neurodegenerative Patterns of Cognitive Clusters of EOAD Subjects: Evidence for Disease Heterogeneity

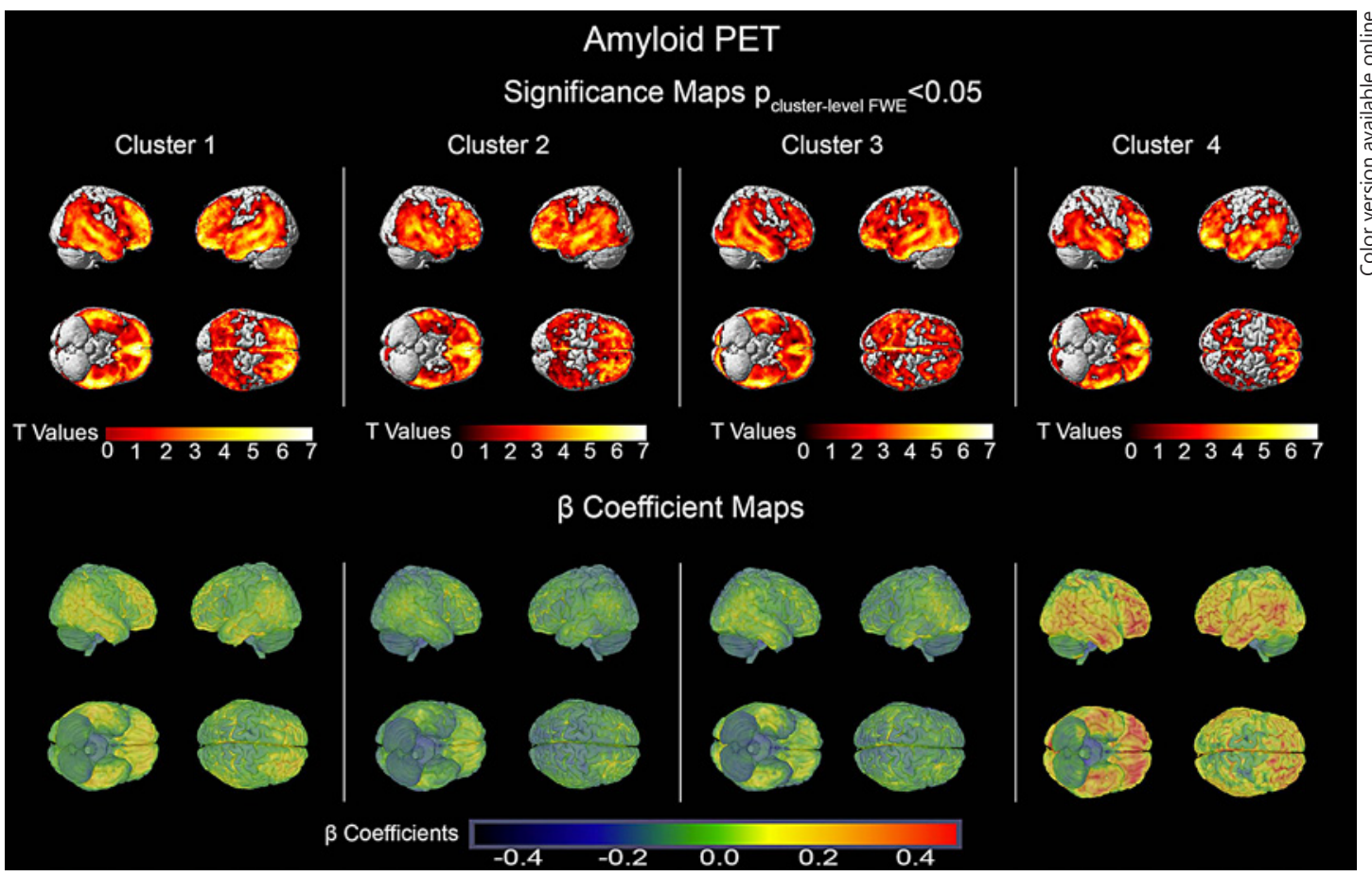

Fig. 3. Amyloid PET significance and $\beta$ coefficient maps showing the comparison of each cluster to $\mathrm{CN}$ participants. The significance maps show $p<0.05$ thresholded FWE cluster-level corrected results. PET, positron emission tomography; FWE, family-wise error.

\section{Longitudinal Cognitive Decline}

Table 1 shows the estimated domain-specific change from baseline to 60 months for subjects in each cognitive cluster. Cluster 1 , who at baseline showed only memory deficits, progressed to develop significant decline in language (overall change -0.84 SD, 95\% CI -1.29 to -0.39 ), visuospatial $(-1.60 \mathrm{SD}, 95 \% \mathrm{CI}-2.26$ to -0.93$)$, and executive skills (6.68 SD, $95 \%$ CI 1.0-2.37) over 5 years. Cluster 2, with baseline deficits in memory and visuospatial function, showed significant improvement of visuospatial function over time (1.53 SD, 95\% CI 0.382.68). Cluster 3 , subjects who had baseline impairments in memory, language, and executive function, showed progressive worsening in their visuospatial skills (-3.11 SD, 95\% CI -4.66 to -1.56). Cluster 4 , which included individuals who already were impaired in all domains, showed a significant decline in language skills ( -4.87 SD, 95\% CI -7.81 to -1.94$)$. The longitudinal changes can be visualized in Figure 4.

We further tested the hypothesis that the EOAD clusters will show differences in their pattern of cognitive change over time. We found no difference in the rates of memory and executive decline over time (Time ${ }^{*}$ cluster interaction $\mathrm{p}_{\text {memory }}=0.15$ and $\mathrm{p}_{\text {executive }}=0.16$ ).

Significant differences in rate of decline were seen for the language $(p=0.013)$ and visuospatial domains $(p<0.001)$. In terms of language function, the overall decline of cluster 4 was significantly greater than the decline observed in other clusters (cluster 4 vs. $1 p=0.008$, cluster 4 vs. $2 p=0.0021$, and cluster 4 vs. $3 p=0.005$ ). The between-clusters significant difference in visuospatial decline was driven by cluster 2 who, interestingly, improved in visuospatial performance over time (cluster 2 vs. $1 p<0.001$, clusters 2 vs. $3 p<0.001$, clusters 2 vs. $4 p=0.0251$ ). 


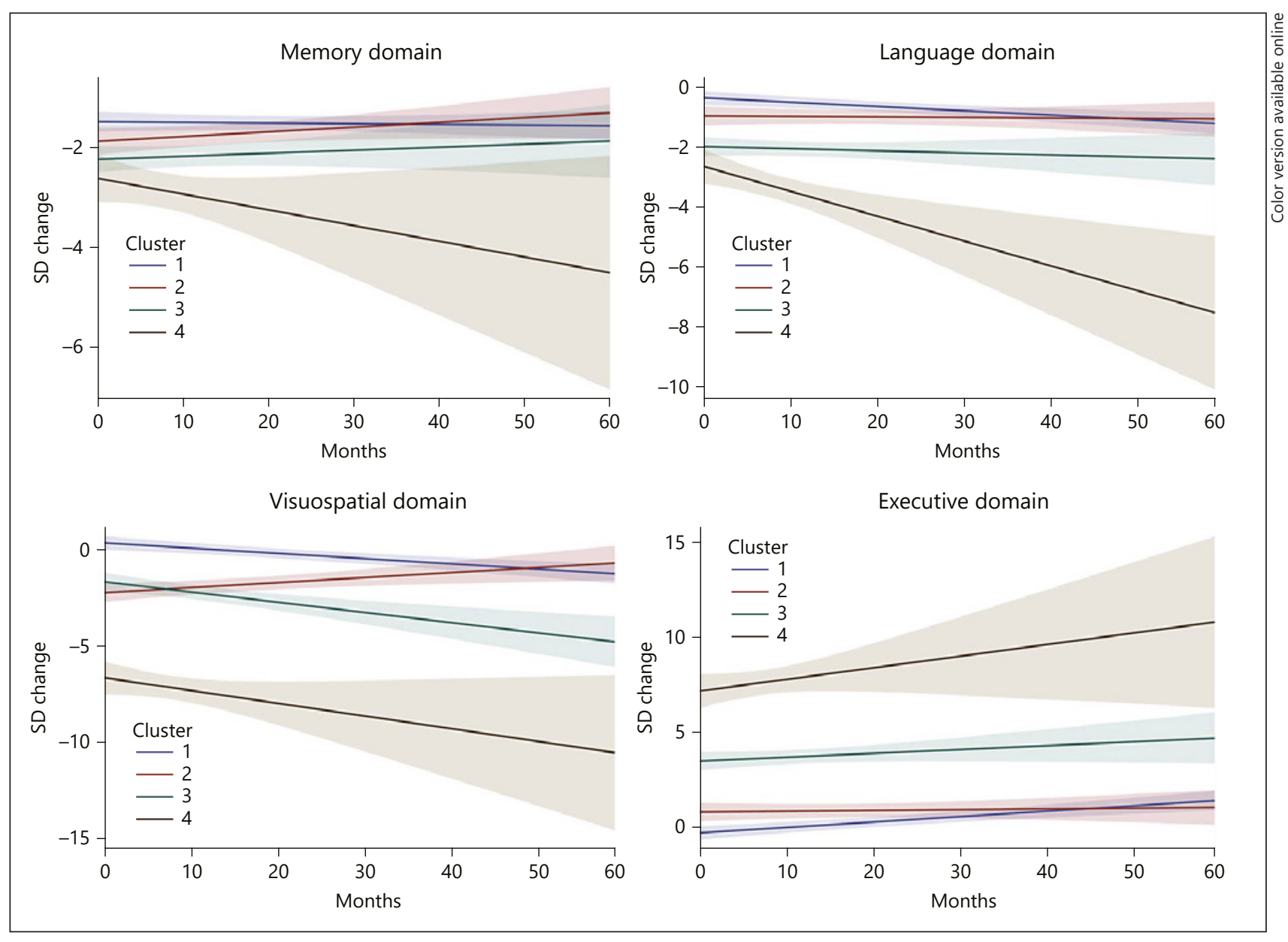

Fig. 4. Longitudinal cognitive decline with $95 \%$ CI by domain. Each figure shows the predicted change in SD over 60 months of each cluster.

\section{Discussion/Conclusion}

We identified several cognitive clusters among amnestic sporadic EOAD participants in the ADNI cohort. This finding agrees with previous work suggesting a heterogeneity among sporadic EOAD patients $[12,48,49]$. We found, in addition to a cluster with a presentation of isolated memory impairment and a globally impaired cluster, two more presentations: one with additional visuospatial impairment and another with additional language + executive dysfunction.

As expected, neurodegenerative changes affected brain regions responsible for the respective cognitive functions that are impaired in each cluster (Fig. 1, 2). Such regional specificity was not observed for brain amyloidosis (Fig. 3). Cluster 1 showed a purely amnestic dysfunction with a corresponding reduction in GMD and glucose metabolism centered in memory regions. The memory and visuospatial deficits of Cluster 2 corresponded to neurodegeneration of the medial temporal and parietal lobes. In Cluster 3, who had affected memory, language, and executive function, the neurodegeneration spread to the memory, language, and executive centers of the brain (medial temporal, lateral temporal, and frontal lobes, respectively; greater in the left hemisphere). Not surprisingly, cluster 4 with global 
Dementia

Cognitive Disorders

\begin{tabular}{l|l}
\hline Dement Geriatr Cogn Disord 2019:48:131-142 \\
\hline DOI: $10.1159 / 000504341$ & $\begin{array}{l}\text { (c) 2020 S. Karger AG, Basel } \\
\text { www.karger.com/dem }\end{array}$ \\
\hline
\end{tabular}

Phillips et al.: Neurodegenerative Patterns of Cognitive Clusters of EOAD Subjects: Evidence for Disease Heterogeneity

cognitive impairment also had global reduction in GMD and glucose metabolism in all cortical regions. Invariably across all clusters, the structural neurodegenerative changes were more extensive compared to the metabolic ones.

Since all participants had an amnestic phenotype, the partially overlapping patterns of neurodegeneration is not surprising. The amnestic AD variant shows the classic neurodegenerative pattern of medial, and in later stages, lateral temporal involvement, followed by parietal, occipital, and lastly frontal lobe changes. The more strikingly different patterns of neurodegeneration such as those seen in logopenic aphasia due to AD and posterior cortical atrophy due to $\mathrm{AD}$ were not observed in this study as subjects with these presentations were explicitly excluded from ADNI.

Longitudinally, we found significant differences between the rate of decline in the language and visuospatial domains. For the language domain, this was primarily due to the significant decline observed in cluster 4 . For the visuospatial domain, it was driven by an overall improvement in visuospatial function that was observed in cluster 2 suggesting that the participants in cluster 2 experienced a learning effect longitudinally. Such improvement has been previously observed by others in a cohort of subjects with mild dementia with a mean age 76.4 years [50]. Overall the data suggest that Cluster 4 has a more aggressive form of EOAD, as none of the other clusters reach the level or pattern of cognitive decline observed in Cluster 4 at baseline, even with a similar disease duration at baseline ( 4.8 years for cluster 4 vs. 4.5-5.8 years for clusters 1-3). Further studies with larger sample size will be needed to better characterize this aggressive variant.

Several strengths and limitations of this study warrant mention. The strengths of our study include the standardization of imaging and neuropsychological data across sites in the ADNI protocol. However, the requirement that all participants have memory impairment limited our ability to study other relatively common nonamnestic EOAD presentations, such as posterior cortical atrophy, logopenic aphasia, or the frontal dysexecutive variant. ADNI employs strict inclusion and exclusion criteria, which means the ADNI sample is not fully representative of all dementia patients. Also as seen in Table 1, there are large betweenclusters differences in the sample sizes, both at baseline and in follow-up. The small sample sizes reduced the power of our analysis, thereby diminishing the ability to present statistically significant differences in the longitudinal decline, both within and between clusters. A large multisite study - the longitudinal early-onset AD study (LEADS, Principal Investigator Liana Apostolova) is currently ongoing. LEADS will prospectively enroll and follow 600 early onset cognitively impaired individuals (CDR 0.5-1) and 100 controls (CDR 0) ages 40-64. LEADS will be able to definitively ascertain whether different patterns of cognitive decline are detectable among study participants.

In conclusion, we found 4 distinct amnestic variants of EOAD suggestive of a high degree of heterogeneity among AD patients in this age group. Larger prospective studies are needed to better characterize these subtypes of sporadic EOAD and delve deeper into any potential environmental, genetic, epigenetic, and pathophysiologic differences that could potentially explain the observed clinical variability.

\section{Acknowledgments}

Data used in preparation of this article were obtained from the ADNI database (adni.loni.usc.edu). As such, the investigators within the ADNI contributed to the design and implementation of ADNI and/or provided data but did not participate in analysis or writing of this report. A complete listing of ADNI investigators can be found at: http://adni.loni.usc.edu/wpcontent/uploads/how_to_apply/ADNI_Acknowledgement_List.pdf. 


\section{Statement of Ethics}

The authors have no ethical conflicts to disclose. The research protocol was reviewed and approved by each site's Institutional Review Board. Informed consent was obtained from all research participants according to the Declaration of Helsinki and the Belmont Report.

\section{Disclosure Statement}

The authors have no conflicts of interest to declare.

\section{Funding Sources}

The analyses reported in this manuscript were funded by the NIA R01 AG040770, NIA K02 AG048240, NIA P30 AG010133, NIA K01 AG049050, NIA R01 AG019771, and the Easton Consortium for Alzheimer's Drug Discovery and Biomarker Development.

Data collection and sharing for this project were funded by the ADNI (National Institutes of Health Grant U01 AG024904) and DOD ADNI (Department of Defense award number W81XWH-12-2-0012). ADNI is funded by the National Institute on Aging, the National Institute of Biomedical Imaging and Bioengineering, and through generous contributions from the following: AbbVie, Alzheimer's Association; Alzheimer's Drug Discovery Foundation; Araclon Biotech; BioClinica, Inc.; Biogen; Bristol-Myers Squibb Company; CereSpir, Inc.; Cogstate; Eisai Inc.; Elan Pharmaceuticals, Inc.; Eli Lilly and Company; EuroImmun; F. Hoffmann-La Roche Ltd and its affiliated company Genentech, Inc.; Fujirebio; GE Healthcare; IXICO Ltd.; Janssen Alzheimer Immunotherapy Research and Development, LLC.; Johnson and Johnson Pharmaceutical Research and Development LLC.; Lumosity; Lundbeck; Merck and Co., Inc.; Meso Scale Diagnostics, LLC.; NeuroRx Research; Neurotrack Technologies; Novartis Pharmaceuticals Corporation; Pfizer Inc.; Piramal Imaging; Servier; Takeda Pharmaceutical Company; and Transition Therapeutics. The Canadian Institutes of Health Research is providing funds to support ADNI clinical sites in Canada. Private sector contributions are facilitated by the Foundation for the National Institutes of Health (www.fnih.org). The grantee organization is the Northern California Institute for Research and Education, and the study is coordinated by the Alzheimer's Therapeutic Research Institute at the University of Southern California. ADNI data are disseminated by the Laboratory for Neuro Imaging at the University of Southern California.

\section{Author Contributions}

M.L.P. contributed to the overall analysis as well as a bulk of the writing. E.C.S., Jr. assisted in running imaging analyses, compiling the data used, and took part in writing/editing of the manuscript. K.A.L. and S.G. completed the statistical analyses and took part in writing/editing of the manuscript. S.L.R. contributed to the preprocessing and data organization of ADNI images and took part in writing/editing of the manuscript. N.G. contributed to the initial analyses by obtaining and organizing all ADNI data and took part in writing/ editing of the manuscript. A.J.S., M.C.C., B.C.D., and G.D.R. are key contributors to many of the ideas presented in this paper and took part in writing/editing of the manuscript. L.G.A. is the senior author who conceptualized this manuscript and participated in all stages of data analyses and writing/editing of the manuscript.

\section{References}

1 Alzheimer's Association. 2017 Alzheimer's disease facts and figures. Alzheimers Dement. 2017;13(4):325-73.

2 Zhu XC, Tan L, Wang HF, Jiang T, Cao L, Wang C, et al. Rate of early onset Alzheimer's disease: a systematic review and meta-analysis. Ann Transl Med. 2015 Mar;3(3):38-38.

3 Koedam EL, Lauffer V, van der Vlies AE, van der Flier WM, Scheltens P, Pijnenburg YA. Early-versus late-onset Alzheimer's disease: more than age alone. J Alzheimers Dis. 2010;19(4):1401-8.

4 Ye BS, Seo SW, Lee Y, Kim SY, Choi SH, Lee YM, et al. Neuropsychological performance and conversion to Alzheimer's disease in early-compared to late-onset amnestic mild cognitive impairment: CREDOS study. Dement Geriatr Cogn Disord. 2012;34(3-4):156-66. 
Phillips et al.: Neurodegenerative Patterns of Cognitive Clusters of EOAD Subjects:

Evidence for Disease Heterogeneity

5 van der Flier WM, Pijnenburg YA, Fox NC, Scheltens P. Early-onset versus late-onset Alzheimer's disease: the case of the missing APOE $\varepsilon 4$ allele. Lancet Neurol. 2011 Mar;10(3):280-8.

6 Mendez MF, Lee AS, Joshi A, Shapira JS. Nonamnestic presentations of early-onset Alzheimer's disease. Am J Alzheimers Dis Other Demen. 2012 Sep;27(6):413-20.

7 Seltzer B, Sherwin I. A comparison of clinical features in early- and late-onset primary degenerative dementia. One entity or two? Arch Neurol. 1983 Mar;40(3):143-6.

8 Loring DW, Largen JW. Neuropsychological patterns of presenile and senile dementia of the Alzheimer type. Neuropsychologia. 1985;23(3):351-7.

9 Filley CM, Kelly J, Heaton RK. Neuropsychologic features of early- and late-onset Alzheimer's disease. Arch Neurol. 1986 Jun;43(6):574-6.

10 Migliaccio R, Agosta F, Possin KL, Canu E, Filippi M, Rabinovici GD, et al. Mapping the Progression of Atrophy in Early- and Late-Onset Alzheimer's Disease. J Alzheimers Dis. 2015;46(2):351-64.

11 Heyman A, Wilkinson WE, Hurwitz BJ, Helms MJ, Haynes CS, Utley CM, et al. Early-onset Alzheimer's disease: clinical predictors of institutionalization and death. Neurology. 1987 Jun;37(6):980-4.

12 Binetti G, Magni E, Padovani A, Cappa SF, Bianchetti A, Trabucchi M. Neuropsychological heterogeneity in mild Alzheimer's disease. Dementia. 1993 Nov-Dec;4(6):321-6.

13 Jacobs D, Sano M, Marder K, Bell K, Bylsma F, Lafleche G, et al. Age at onset of Alzheimer's disease: relation to pattern of cognitive dysfunction and rate of decline. Neurology. 1994 Jul;44(7):1215-20.

14 Koss E, Edland S, Fillenbaum G, Mohs R, Clark C, Galasko D, et al. Clinical and neuropsychological differences between patients with earlier and later onset of Alzheimer's disease: A CERAD analysis, Part XII. Neurology. 1996 Jan;46(1):136-41.

15 Fujimori M, Imamura T, Yamashita H, Hirono N, Ikejiri Y, Shimomura T, et al. Age at onset and visuocognitive disturbances in Alzheimer disease. Alzheimer Dis Assoc Disord. 1998 Sep;12(3):163-6.

16 Imamura T, Takatsuki Y, Fujimori M, Hirono N, Ikejiri Y, Shimomura T, et al. Age at onset and language disturbances in Alzheimer's disease. Neuropsychologia. 1998 Sep;36(9):945-9.

17 Mendez MF, Ghajarania M, Perryman KM. Posterior cortical atrophy: clinical characteristics and differences compared to Alzheimer's disease. Dement Geriatr Cogn Disord. 2002;14(1):33-40.

18 Mendez MF. Early-onset Alzheimer's disease: nonamnestic subtypes and type 2 AD. Arch Med Res. 2012 Nov; 43(8):677-85.

19 Ho GJ, Hansen LA, Alford MF, Foster K, Salmon DP, Galasko D, et al. Age at onset is associated with disease severity in Lewy body variant and Alzheimer's disease. Neuroreport. 2002 Oct;13(14):1825-8.

20 Barclay LL, Zemcov A, Blass JP, McDowell FH. Factors associated with duration of survival in Alzheimer's disease. Biol Psychiatry. 1985 Jan;20(1):86-93.

21 Mortimer JA, Ebbitt B, Jun SP, Finch MD. Predictors of cognitive and functional progression in patients with probable Alzheimer's disease. Neurology. 1992 Sep;42(9):1689-96.

22 Rossor MN, Fox NC, Mummery CJ, Schott JM, Warren JD. The diagnosis of young-onset dementia. Lancet Neurol. 2010 Aug;9(8):793-806.

23 Warren JD, Fletcher PD, Golden HL. The paradox of syndromic diversity in Alzheimer disease. Nat Rev Neurol. 2012 Aug;8(8):451-64.

24 Kim SH, Seo SW, Yoon DS, Chin J, Lee BH, Cheong HK, et al. Comparison of neuropsychological and FDG-PET findings between early- versus late-onset mild cognitive impairment: A five-year longitudinal study. Dement Geriatr Cogn Disord. 2010;29(3):213-23.

25 Szigeti K, Doody RS. Should EOAD patients be included in clinical trials? Alzheimers Res Ther. 2011 Feb;3(1): 4.

26 Petersen RC. Mild cognitive impairment. Continuum: Lifelong Learning in Neurology. 2007;13:15-38.

27 Petersen RC, Aisen PS, Beckett LA, Donohue MC, Gamst AC, Harvey DJ, et al. Alzheimer's Disease Neuroimaging Initiative (ADNI): clinical characterization. Neurology. 2010 Jan;74(3):201-9.

28 Petersen RC, Smith GE, Waring SC, Ivnik RJ, Tangalos EG, Kokmen E. Mild cognitive impairment: clinical characterization and outcome. Arch Neurol. 1999 Mar;56(3):303-8.

29 McKhann G, Drachman D, Folstein M, Katzman R, Price D, Stadlan EM. Clinical diagnosis of Alzheimer's disease: report of the NINCDS-ADRDA Work Group under the auspices of Department of Health and Human Services Task Force on Alzheimer's Disease. Neurology. 1984 Jul;34(7):939-44.

30 Dubois B, Feldman HH, Jacova C, Dekosky ST, Barberger-Gateau P, Cummings J, et al. Research criteria for the diagnosis of Alzheimer's disease: revising the NINCDS-ADRDA criteria. Lancet Neurol. 2007 Aug;6(8):734-46.

31 Jack CR Jr, Albert MS, Knopman DS, McKhann GM, Sperling RA, Carrillo MC, et al. Introduction to the recommendations from the National Institute on Aging-Alzheimer's Association workgroups on diagnostic guidelines for Alzheimer's disease. Alzheimers Dement. 2011 May;7(3):257-62.

32 Wyman BT, Harvey DJ, Crawford K, Bernstein MA, Carmichael O, Cole PE, et al.; Alzheimer's Disease Neuroimaging Initiative. Standardization of analysis sets for reporting results from ADNI MRI data. Alzheimers Dement. 2013 May; $9(3): 332-7$.

33 Jack CR Jr, Bernstein MA, Borowski BJ, Gunter JL, Fox NC, Thompson PM, et al.; Alzheimer's Disease Neuroimaging Initiative. Update on the magnetic resonance imaging core of the Alzheimer's disease neuroimaging initiative. Alzheimers Dement. 2010 May;6(3):212-20.

34 Jack CR Jr, Bernstein MA, Fox NC, Thompson P, Alexander G, Harvey D, et al. The Alzheimer's Disease Neuroimaging Initiative (ADNI): MRI methods. J Magn Reson Imaging. 2008 Apr;27(4):685-91. 
35 Whitwell JL. Voxel-based morphometry: an automated technique for assessing structural changes in the brain. J Neurosci. 2009 Aug;29(31):9661-4.

36 Risacher SL, Kim S, Shen L, Nho K, Foroud T, Green RC, et al.; Alzheimer's Disease Neuroimaging Initiative (ADNI). The role of apolipoprotein E (APOE) genotype in early mild cognitive impairment (E-MCI). Front Aging Neurosci. 2013 Apr;5:11.

37 Risacher SL, Saykin AJ, West JD, Shen L, Firpi HA, McDonald BC; Alzheimer's Disease Neuroimaging Initiative (ADNI). Baseline MRI predictors of conversion from MCI to probable AD in the ADNI cohort. Curr Alzheimer Res. 2009 Aug;6(4):347-61.

38 Risacher SL, Shen L, West JD, Kim S, McDonald BC, Beckett LA, et al.; Alzheimer's Disease Neuroimaging Initiative (ADNI). Longitudinal MRI atrophy biomarkers: relationship to conversion in the ADNI cohort. Neurobiol Aging. 2010 Aug;31(8):1401-18.

39 Jagust WJ, Bandy D, Chen K, Foster NL, Landau SM, Mathis CA, et al.; Alzheimer's Disease Neuroimaging Initiative. The Alzheimer's Disease Neuroimaging Initiative positron emission tomography core. Alzheimers Dement. 2010 May;6(3):221-9.

40 Jagust WJ, Landau SM, Koeppe RA, Reiman EM, Chen K, Mathis CA, et al. The Alzheimer's Disease Neuroimaging Initiative 2 PET Core: 2015. Alzheimers Dement. 2015 Jul;11(7):757-71.

41 Wechsler D. Wechsler memory scale-revised (WMS-R). Psychological Corporation; 1987.

42 Rey A. L'examen clinique en psychologie [The clinical psychological examination]Paris: Presses Universitaires de France; 1964.

43 Kaplan E, Goodglass H, Weintraub S. The Boston naming test. Philadelphia: Lea \& Febiger; 1983.

44 Butters N, Granholm E, Salmon DP, Grant I, Wolfe J. Episodic and semantic memory: a comparison of amnesic and demented patients. J Clin Exp Neuropsychol. 1987 Oct;9(5):479-97.

45 Goodglass H, Kaplan E. The assesment of aphasia and related disorders. 2nd ed. Philadelphia: Lea \& Febiger; 1983.

46 Reitan RM. Validity of the Trail Making Test as an indicator of organic brain damage. Percept Mot Skills. 1958; 8(3):271-6.

47 Ward JH Jr. Hierarchical Grouping to Optimize an Objective Function. J Am Stat Assoc. 1963;58(301):236-44.

48 Lehmann M, Ghosh PM, Madison C, Laforce R Jr, Corbetta-Rastelli C, Weiner MW, et al. Diverging patterns of amyloid deposition and hypometabolism in clinical variants of probable Alzheimer's disease. Brain. 2013 Mar; 136(Pt 3):844-58.

49 Ossenkoppele R, Cohn-Sheehy BI, La Joie R, Vogel JW, Möller C, Lehmann M, et al. Atrophy patterns in early clinical stages across distinct phenotypes of Alzheimer's disease. Hum Brain Mapp. 2015 Nov;36(11):442137.

50 Cacho J, García-García R, Fernández-Calvo B, Gamazo S, Rodríguez-Pérez R, Almeida A, et al. Improvement pattern in the clock drawing test in early Alzheimer's disease. Eur Neurol. 2005;53(3):140-5. 